\title{
Correction to: DNA barcoding of Oryza: conventional, specific, and super barcodes
}

\author{
Wen Zhang ${ }^{1,2} \cdot$ Yuzhe Sun $^{1,2} \cdot$ Jia Liu $^{1,4} \cdot$ Chao Xu$^{1} \cdot$ Xinhui Zou $^{1} \cdot$ Xun Chen ${ }^{1,3} \cdot$ Yanlei Liu $^{1,2} \cdot$ Ping Wu $^{1,2}$. \\ Xueying Yang ${ }^{5}$. Shiliang Z Zou ${ }^{1,2}$ (1)
}

Published online: 15 December 2020

(C) The Author(s) 2020

\section{Correction to: Plant Molecular Biology https://doi.org/10.1007/s11103-020-01054-3}

In the above mentioned publication the funding section was missing. The original article has been corrected and the funding section is also published here.

Funding This study was partly supported by the Strategic Priority Research Program of the Chinese Academy of Sciences, Grant No. XDA 19050303 \& XDA 23080204, and the Fundamental Research Funds for the Central Public-Service Research Institute [2018JB001].

Publisher's Note Springer Nature remains neutral with regard to jurisdictional claims in published maps and institutional affiliations.

The original article can be found online at https://doi.org/10.1007/ s11103-020-01054-3.

Xueying Yang

yxystyhhp@163.com

$\triangle$ Shiliang Zhou

slzhou@ibcas.ac.cn

1 State Key Laboratory of Systematic \& Evolutionary

Botany, Institute of Botany, Chinese Academy of Sciences,

Beijing 100093, China

2 College of Life Sciences, University of Chinese Academy of Sciences, Beijing 100049, China

3 College of Landscape Architecture, Northeast Forestry University, Haerbin 150040, China

4 College of Life Science, Sichuan Agricultural University, Yaan 625014, Sichuan, China

5 Key Laboratory of Forensic Genetics, Institute of Forensic Science, Ministry of Public Security, China, Beijing 100038, China 